\title{
Endovascular Treatment of Intracranial Aneurysms Associated with Brain Arteriovenous Malformations
}

\author{
Boeun Lee ${ }^{1}$, Jae Whan Lee ${ }^{1}$, Keun Young Park ${ }^{1}$, Dong Joon Kim ${ }^{2}$, Byung Moon Kim ${ }^{2}$, Joonho Chung ${ }^{1,3}$ \\ ${ }^{1}$ Department of Neurosurgery, Severance Hospital, Yonsei University College of Medicine, Seoul, Korea \\ ${ }^{2}$ Department of Radiology, Severance Hospital, Yonsei University College of Medicine, Seoul, Korea \\ ${ }^{3}$ Severance Institute for Vascular and Metabolic Research, Yonsei University College of Medicine, Seoul, Korea
}

Received: June 12, 2019

Accepted: August 19, 2019

Corresponding Author:

Joonho Chung, M.D., Ph.D.

Department of Neurosurgery,

Severance Hospital Severance

Institute for Vascular and

Metabolic Research Yonsei

University College of Medicine

50-1, Yonsei-ro, Seodaemun-gu,

Seoul 03722, Korea

Tel: $+82-2-2228-2150$

Fax: $+82-2-393-9979$

E-mail: ns.joonho.chung@gmail.com

\section{Objective}

Brain arteriovenous malformation (AVM) is frequently associated with the presence of intracranial aneurysms, which increase hemorrhage rates resulting in unfavorable outcomes. We report our experience with endovascular treatment (EVT) of intracranial aneurysms associated with AVM.

\section{Methods}

Between March 2010 and February 2017, we treated 274 patients with AVMs. Among them, 27 (9.9\%) patients with intracranial aneurysms associated with AVM were treated endovascularly. Clinical and radiographic data for these patients were retrospectively reviewed.

Results

We treated 32 aneurysms ( 10 intranidal and 22 proximal aneurysms) in 27 patients. The most frequent presenting symptoms were hemorrhage (59.3\%) caused by an AVM nidus in 7 cases and by aneurysm rupture in 9 cases. Of 22 proximal flow-related aneurysms, we performed a simple coiling technique in 16 aneurysms and 6 parent artery occlusions. For 10 intranidal aneurysms, we performed targeted embolization with liquid embolic materials, such as n-Butyl cyanoacrylate or Onyx. There were three procedure-related complications and only one patient suffered transient hemiparesis. Favorable outcome (modified Rankin Scale, mRS 0-2) was achieved in 18 (66.7\%) patients and unfavorable outcome (mRS 3-6) in 9 (33.3\%) patients, including one death.

Conclusion

When facing intracranial aneurysms associated with AVMs, EVT might be a useful treatment option for proximal and intranidal aneurysms.

Keywords: Brain arteriovenous malformation; Endovascular treatment; Flow-related aneurysm; Intranidal aneurysm

\section{INTRODUCTION}

Brain arteriovenous malformations (AVMs) are complex vas- cular lesions that consist of abnormal connections between cerebral arteries and veins without an associated capillary bed. AVM patients can present with hemorrhage, seizure, neurological defi-

Copyright (C) 2019 The Korean Neurointensive Care Society

This is an Open Access article distributed under the terms of the Creative Commons Attribution Non-Commercial License (http://creativecommons.org/licenses/by-nc/4.0/) which permits unrestricted non-commercial use, distribution, and reproduction in any medium, provided the original work is properly cited. 
cit, or headache, and AVMs often remain unrecognized until they become symptomatic. With a prevalence that is likely less than 10 per 100000, they are relatively rare, but can nevertheless have devastating consequences for patients who harbor them. Once an AVM ruptures, the risk of additional hemorrhage over the first year increases to $6 \%$ to $18 \% 3-7$ ), before returning to $2 \%$ to $4 \%$ per year, which is the hemorrhage rate of previously diagnosed unruptured AVMs. An increased risk for hemorrhage has been reported in previous studies when an AVM is associated with an aneurysm. The association of intracranial aneurysms with AVMs has been well-established. Depending on the case series, aneurysms are present in 3-58\% of patients with AVMs ${ }^{8,10,13,16)}$.

Over the last decade, outstanding progress in endovascular techniques targeting intracerebral aneurysm repairs has highlighted the need for preventive AVMs treatments in the context of associated aneurysms, which is a growing issue. Treating either AVM or aneurysms is challenging. Some experts have recommended early treatment of aneurysms, but some have recommended treatment of the symptomatic lesion first. In terms of treating both lesions simultaneously, EVT may be preferable. Thus, we report our experience with endovascular treatment (EVT) for intracranial aneurysms associated with AVMs.

\section{MATERIALS AND METHODS}

This retrospective study was approved by the Institutional Review Board and the requirement for informed consent was waived. Between March 2010 and February 2017, we treated 274 patients with brain AVMs. Among them, a total of 27 (9.9\%) patients who met the following criteria were included in this study: 1) patients diagnosed with intracranial aneurysms associated with AVMs by computed tomography (CT), magnetic resonance imaging (MRI), or digital subtraction angiography (DSA); and 2) aneurysms that were treated by EVT. Cerebral aneurysms associated with AVMs were defined and divided into two categories; intranidal aneurysms (i.e., aneurysms located within or in the immediate vicinity of the AVM nidus) and proximal flow-related aneurysms (i.e., aneurysms arising along the course of arteries that eventually supply the AVM). Clinical and radiographic data for these patients were retrospectively reviewed. All patients underwent DSA with 3-dimensional rotational angiography to evaluate vascular architecture and the location of their lesions.

Treatment decisions were made by agreement of microvascular neurosurgeons and neurointerventionists. All procedures were performed under general anesthesia with a standard approach from the common femoral artery. A 6-Fr femoral sheath was inserted into the common femoral artery. A 5-Fr or 6-Fr guiding catheter
Envoy (Codman Neurovascular, Miami Lakes, FL, USA) was positioned in the ICA or VA. Intravenous systemic heparin $(50 \mathrm{U} / \mathrm{kg})$ was administered while placing the guiding catheter. A microcatheter was advanced super-selectively to the AVM's small feeding arteries. For intranidal aneurysms, n-Butyl cyanoacrylate (NBCA) or Onyx were used to occlude the aneurysms and the nidus simultaneously with the intention of reducing hemorrhagic complications that could arise during the procedure interval. For proximal flow-related aneurysms, a variety of techniques were performed as needed: simple coiling, multiple-microcatheter, balloon remodeling, or the stent-assisted technique as a reconstruction technique, and deconstruction techniques, such as parent artery occlusion.

Procedure-related events were defined as any events during the procedure that were symptomatic or asymptomatic. Clinical outcomes were assessed using the modified Rankin Scale (mRS) and evaluated at the time of discharge and out-patient follow-up by an independent investigator who was not involved in patient care. Angiographic and clinical follow-ups were performed at 6 to 12 months in all patients.

\section{RESULTS}

Demographic and morphologic characteristics of the 27 patients with intracranial aneurysms associated with AVMs are summarized in Table 1. There were 9 women (33.3\%) and 18

Table 1. Demographic and morphological characteristics for 27 patients with cerebral aneurysms associated with arteriovenous malformations.

\begin{tabular}{lc}
\hline Aneurysms associated with arteriovenous malformations $(\mathrm{N}=27)$ \\
\hline Age at presentation, $\mathrm{y}($ mean $\pm \mathrm{SD})$ & $38.7 \pm 12.6$ \\
Female, $\mathrm{n}(\%)$ & $9(33.3)$ \\
Presentations, $\mathrm{n}(\%)$ & $16(59.3)$ \\
$\quad$ Hemorrhage & $11(40.7)$ \\
$\quad$ Non-hemorrhage & \\
Locations, $\mathrm{n}(\%)$ & $23(85.2)$ \\
$\quad$ Supratentorial & $4(14.8)$ \\
Infratentorial & \\
AVM size, $\mathrm{n}(\%)$ & $7(25.9)$ \\
$\quad<3 \mathrm{~cm}$ & $17(63.0)$ \\
$3-6 \mathrm{~cm}$ & $3(11.1)$ \\
$>6 \mathrm{~cm}$ & \\
Venous drainage pattern, $\mathrm{n}(\%)$ & $4(14.8)$ \\
Deep venous drainage only & $5(18.5)$ \\
Any deep venous drainage & \\
Associated arterial aneurysm, $\mathrm{n}(\%)$ & $5(18.5)$ \\
Intranidal & $17(63.0)$ \\
Proximal & $5(18.5)$ \\
Intranidal \& Proximal & \\
\hline
\end{tabular}


men $(66.7 \%)$. Patient ages ranged from 18 to 61 years, with a mean age of 38.7 years. Four (14.8\%) AVMs were located in an infratentorial location and 23 (85.2\%) were supratentorial. Sixteen $(59.3 \%)$ patients initially presented with hemorrhage. The remaining 11 patients (40.7\%) initially presented with symptoms or signs unrelated to hemorrhage. Among the patients who presented with hemorrhage, bleeding focus was determined by the location and distribution of the hematoma on enhanced CT scan and this was correlated with DSA. Bleeding was caused by the AVM nidus in 7 cases and by aneurysm rupture in 9 cases. Eleven patients had no history of hemorrhage.

Five patients had AVMs with multiple arterial aneurysms, thus there was a total of 32 aneurysms in 27 patients. The associated aneurysms were divided into 22 (68.8\%) proximal flow-related aneurysms and $10(31.3 \%)$ intranidal aneurysms. For 22 proximal flow-related aneurysms, we performed a simple coiling technique in 16 aneurysms and parent artery occlusion (PAO) in 6. For 10 intranidal aneurysms, we performed targeted embolization with liquid embolic materials (i.e., NBCA or Onyx). Ten patients underwent microsurgical nidus removal and 12 patients underwent gamma knife surgery after embolization. The other five AVMs were completely occluded by EVT simultaneously with the aneurysms.

There were three procedure-related complications and only one patient suffered transient hemiparesis. Favorable outcome (mRS $0-2$ ) was achieved in 18 (69.2\%) and unfavorable outcome (mRS $3-6)$ in $8(30.8 \%)$ patients, including one death at discharge.

\section{Case Illustrations}

\section{Case 1}

A 54-year-old female was admitted to our institute with deep drowsy consciousness and right hemiparesis. Brain CT scan revealed diffuse subarachnoid hemorrhage in the basal cistern, quadrigeminal cistern, and intraparenchymal hemorrhage in the left pons-to-cerebellum and the left occipital lobe (Fig. 1A). DSA revealed a proximal flow-related aneurysm in the $\mathrm{P} 3$ segment of the left posterior cerebral artery (PCA) and a $2.7 \times 2.5 \mathrm{~mm}$ sized AVM nidus in the left occipital lobe fed by the left middle cerebral artery (MCA), left anterior cerebral artery (ACA), and the left PCA (Fig. 1B). It drained into cortical veins connected to the superior sagittal sinus. An intraparenchymal hemorrhage in the left pons implied a P3 segment aneurysm rupture and the aneurysm showed a fusiform-like wide-neck involving 360-degrees of the PCA. PAO with coiling was performed to occlude the ruptured aneurysm and one of the feeding arteries into the AVM nidus (Fig. 1C). Then, the left MCA and ACA were super-selected by Apollo detachable-tip microcatheters (Medtronic, Irvine, CA) and Onyx embolization was performed (Fig. 1D). Partial occlusion of the AVM nidus let us perform additional microsurgical nidus removal. The nidus filled with Onyx guided us to delineate the margin of the AVM nidus and helped us remove the nidus completely (Fig. 1E). At one-month follow-up, a CT scan revealed a post-treatment coil mass and empty space of the nidus (Fig. 1F). However, the patient's clinical outcome was an unfavorable, $\mathrm{mRS}$ of 4 at her discharge.

\section{DISCUSSION}

It has been established that hemorrhage is the most devastating AVM complication and the annual risk of rupture, regardless of its characteristics, is $4 \%$ on average ${ }^{1)}$. However, AVM may have different behaviors due to the following factors: elapsed time after diagnosis, presence of deep venous drainage, associated aneurysms, AVM location, size, male sex, age, and other variables specific to each patient. The hemorrhage risk if there are associated aneurysms has been reported as $7 \%$ per year and most hemorrhages are equally distributed between aneurysms and AVMs, which means an aneurysm may be the site of rupture ${ }^{1}$. The reported rate of intracranial aneurysms associated with AVMs varies widely in previously published data sets, depending on the definition of what represents an associated aneurysm. Intracranial aneurysms associated with AVMs may be caused by hemodynamic stress related to the AVM or they may be congenital ${ }^{14,20)}$. The hemodynamic stress secondary to increased AVM blood flow plays a significant role in provoking this coexistence of lesions. Patients with subarachnoid hemorrhage, intraparenchymal hemorrhage, or both and an angiographically demonstrated AVM with an associated aneurysm, should have therapy directed first toward the lesion that is suspected to be the source of the hemorrhage. In the present study, 32 aneurysms associated with AVMs in 27 patients were treated by EVT with a relatively low complication rate. Twenty-two proximal flow-related aneurysms were treated by simple coiling or PAO, whereas 10 intranidal aneurysms were treated by targeted embolization with liquid embolic materials. Favorable outcome was achieved in $70 \%$ of 27 patients including 16 patients with hemorrhage. EVT may be considered a useful treatment for aneurysms associated with AVMs. However, our results should not alter the fundamental role of EVT in the management of AMVs, especially as an adjuvant to microsurgery and radiosurgery.

The vascular architecture of an AVM is an important consideration when planning treatment. Along with a thorough clinical examination, all AVM patients need detailed preoperative radiographic clarification of their AVM's anatomy, architecture, and associated aneurysms obtained with MRI and high-frame rate 

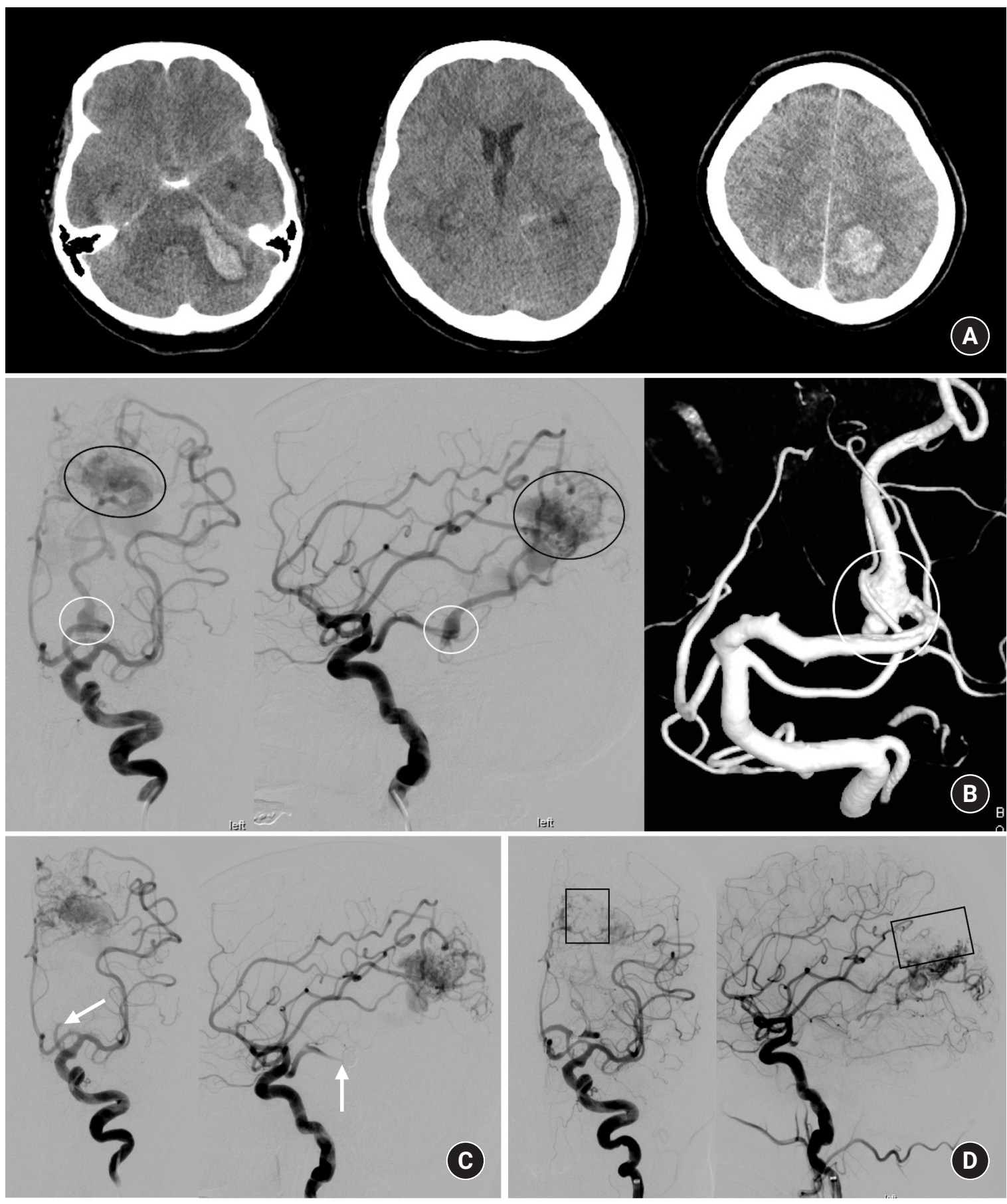

Fig. 1. A 54-year-old female was admitted to our institute with deep drowsy consciousness and right hemiparesis. (A) Brain computed tomography scan revealed diffuse subarachnoid hemorrhage in the basal cistern and quadrigeminal cistern and intraparenchymal hemorrhage in the left pons-to-cerebellum and left occipital lobe. (B) Digital subtraction angiography revealed a proximal flow-related aneurysm on the P3 segment of the left posterior cerebral artery (PCA) and a $2.7 \times 2.5 \mathrm{~mm}$ sized arteriovenous malformation (AVM) nidus in the left occipital lobe fed by the left middle cerebral artery (MCA), left anterior cerebral artery (ACA), and the left PCA. It drained into the cortical veins-to-superior sagittal sinus. (C) Parent artery occlusion with coiling was performed to occlude the ruptured aneurysm and one of the feeding arteries to the AVM nidus. (D) Left MCA and ACA were super-selected by detachable-tip microcatheters and Onyx embolization was performed. (E) Partial occlusion of the AVM nidus let us perform additional microsurgical nidus removal. The nidus filled with Onyx guided us to delineate the margin of the AVM nidus and helped us remove the nidus completely. (F) One-months follow-up CT scan revealed a post-treatment coil mass and empty space of the nidus. White circle indicates a ruptured aneurysm associated AVM. Black circle indicates the AVM nidus. White arrow indicates the P3 segment occlusion by coiling. Rectangle indicates the partially occluded nidus by Onyx embolization. (Continued to the next page) 

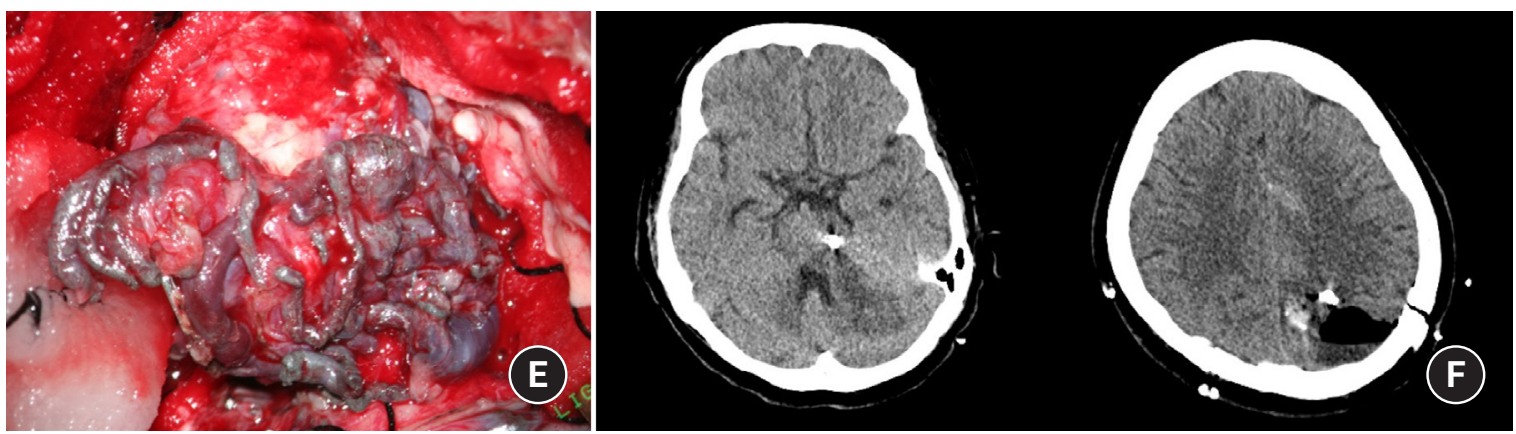

Fig. 1. (Continued from the previous page) A 54-year-old female was admitted to our institute with deep drowsy consciousness and right hemiparesis. (A) Brain computed tomography scan revealed diffuse subarachnoid hemorrhage in the basal cistern and quadrigeminal cistern and intraparenchymal hemorrhage in the left pons-to-cerebellum and left occipital lobe. (B) Digital subtraction angiography revealed a proximal flowrelated aneurysm on the P3 segment of the left posterior cerebral artery (PCA) and a $2.7 \times 2.5 \mathrm{~mm}$ sized arteriovenous malformation (AVM) nidus in the left occipital lobe fed by the left middle cerebral artery (MCA), left anterior cerebral artery (ACA), and the left PCA. It drained into the cortical veins-to-superior sagittal sinus. (C) Parent artery occlusion with coiling was performed to occlude the ruptured aneurysm and one of the feeding arteries to the AVM nidus. (D) Left MCA and ACA were super-selected by detachable-tip microcatheters and Onyx embolization was performed. (E) Partial occlusion of the AVM nidus let us perform additional microsurgical nidus removal. The nidus filled with Onyx guided us to delineate the margin of the AVM nidus and helped us remove the nidus completely. (F) One-months follow-up CT scan revealed a posttreatment coil mass and empty space of the nidus. White circle indicates a ruptured aneurysm associated AVM. Black circle indicates the AVM nidus. White arrow indicates the P3 segment occlusion by coiling. Rectangle indicates the partially occluded nidus by Onyx embolization.

DSA. The angiography must delineate the characteristics of the AVM, including its arterial feeders, venous drainage, internal angioarchitecture, and associated vascular lesions, and it must define the collateral circulation and venous drainage pathways of the normal brain tissue. Treatment decisions should be considered to maximize clinical outcomes and to minimize risks, and can be made regarding the best management approach by comparing the natural history of the lesion with known associated intervention-related morbidity and mortality ${ }^{11)}$. However, treatment guidelines for brain AVMs are still controversial despite outstanding advances in diagnostic and therapeutic resources.

Driven largely by innovations in devices and techniques, the field of neurointerventional surgery is constantly changing. As these changes have occurred across the board, EVT for brain AVMs has seen corresponding advances. Innovations defined by the improvements seen in newer and better embolic materials, microcatheters (flow-directed microcatheters, Onyx comparable and dual lumen balloon microcatheters, and detachable-tip microcatheters), and other devices (distal access intermediate catheters) have resulted in safer and more effective embolization of cerebral $\mathrm{AVMs}^{2}$. EVT can be applied to AVM treatment in various ways: preoperative, intraoperative, pre-radiosurgery, curative, palliative, or post-radiosurgery ${ }^{9,18)}$.

Thus, goals should be defined prior to each procedure. Additionally, traumatic super-selective microcatheterization is a key. In the setting of AVM EVT, all AVM locations should be regarded as eloquent ${ }^{18)}$. Recently, AVM EVT has been used as a curative option that is mainly done in an adjuvant manner, although these advances have made stand-alone embolization a possibility in many patients. EVT of aneurysm-associated AVMs is beneficial before microsurgery or radiosurgery. Thus, physician experience with treating AVMs includes being capable of performing microsurgery and radiosurgery in addition to EVT, with concordant facilities.

Anatomic cures can be accomplished by a combination of two or more modalities, one of which is usually EVT. A multimodality treatment plan can minimize risk and improve patient outcomes. As necessary integration of different treatment modalities, such as microsurgery, radiosurgery, and EVT, increases cure rates tend to be higher. Especially, explosive progress in endovascular devices and techniques provides better quality options for EVT performed alone as well as an adjuvant treatment before microsurgery or a concurrent therapy with radiosurgery. Preoperative embolization reduced mean nidus volume by $74 \%$ to $84 \%^{19)}$. However, pre- or post-radiosurgery embolization to where less likely respond to radiation, to high-flow fistulas or to associated aneurysms consists of conflicting evidence. As physicians have gained more experience with EVT, curative embolization rate is increasing ${ }^{12,15,17)}$.

There are several limitations to this study. This study was a retrospective study with a small case series so that the effects of possible selection bias cannot be excluded. In addition, it was difficult to have a true control group for evaluating the safety and efficacy of EVT with associated aneurysms. Furthermore, a longer 
follow-up period is needed to evaluate the procedure's true safety and efficacy.

\section{CONCLUSION}

When facing intracranial aneurysms associated with AVMs, EVT might be a useful treatment option for proximal flow-related and intranidal aneurysms. This procedure could cure aneurysms and AVMs simultaneously, or be good for adjuvant treatment before microsurgical nidus removal or gamma knife surgery for AVMs.

\section{NOTES}

\section{Conflict of interest}

We declare that we have no conflict of interest.

\section{Ethical approval}

all human subjects study procedures were in accordance with the ethical standards of our Institutional Review Board and the 1964 Declaration of Helsinki and its later amendments or comparable ethical standards.

\section{Informed consent}

In this retrospective study, the requirement for informed consent was waived.

\section{Funding}

No funding was received for this study.

\section{REFERENCES}

1. Brown RD, Wiebers DO, Forbes GS. Unruptured intracranial aneurysms and arteriovenous malformations: frequency of intracra- nial hemorrhage and relationship of lesions. J Neurosurg 1990;73:859-863.

2. Crowley RW, Ducruet AF, McDougall CG, Albuquerque FC. Endovascular advances for brain arteriovenous malformations. Neurosurgery 2014;74:S74-S82.

3. da Costa L, Thines L, Dehdashti AR, Wallace MC, Willinsky RA, Tymianski M, et al. Managementandclinical outcome of posterior fossa arteriovenous malformations: report on a single-centre 15-year experience. J Neurol Neurosurg Psychiatry 2009;80:376-379.

4. Elhammady MS, Aziz-Sultan MA, Heros RC. The management of cerebral arteriovenous malformations associated with aneurysms. World Neurosurg 2013;80:e123-9.
5. Flores BC, Klinger DR, Rickert KL, Barnett SL, Welch BG, White JA, et al. Management of intracranial aneurysms associated with arteriovenous malformations. Neurosurg Focus 2014;37:E11.

6. Garcia-Monaco R, Rodesch G, Alvarez H, Lizuka Y, Hui F, Lasjaunias P. Pseudoaneurysms within ruptured intracranial arteriovenous malformations: diagnosis and early endovascular management. AJNR Am J Neuroradiol 1993;14:315-321.

7. Gross BA, Du R. Natural history of cerebral arteriovenous malformations: a meta analysis. J Neurosurg 2013;118:437-443.

8. Lv X, Wu Z, Li Y, Yang X, Jiang C, Sun Y, et al. Endovascular treatment of cerebral aneurysms associated with arteriovenous malformatioins. Eur J Radiol 2012;81:1296-1298.

9. Martin NA, Khanna R, Doberstein C, Bentson J. Therapeutic embolization of arteriovenous malformations: the case for and against. Clin Neurosurg 2000;46:295-318.

10. Meisel HJ, Mansmann U, Alvarez H, Rodesch G, Brock M, Lasjaunias P. Cerebral arteriovenous malformations and associated aneurysms: analysis of 305 cases from a series of 662 patients. Neurosurgery 2000;46:793-802.

11. Novakovic RL, Lazzaro MA, Castonguay AC, Zaidat OO. The diagnosis and management of brain arteriovenous malformations. Neurol Clin 2013;31:749-763.

12. Pierot L, Cognard C, Herbreteau D, Fransen H, van Rooij WJ, Boccardi E, et al. Endovascular treatment of brain arteriovenous malformations using a liquid embolic agent: results of a prospective, multicenter study (BRAVO). Eur Radiol 2013;23:28382845.

13. Piotin M, Ross IB, Weill A, Kothimbakam R, Moret J. Intracranial arterial aneurysms associated with arteriovenous malformations: endovascular treatment. Radiology 2001;220:506-513.

14. Redekop G, TerBrugge K, Montanera W, Willinsky R. Arterial aneurysms associated with cerebral arteriovenous malformations: classification, incidence, and risk of hemorrhage. J Neurosurg 1998;89:539-546.

15. Renieri L, Consoli A, Scarpini G, Grazzini G, Nappini S, Mangiafico S. Double arterial catheterization technique for embolization of brain arteriovenous malformations with onyx. Neurosurgery 2013;72:92-98.

16. Stapf C, Mast H, Sciacca RR, Choi JH, Khaw AV, Connolly ES, et al. Predictors of hemorrhage in patients with untreated brain arteriovenous malformation. Neurology 2006;66:1350-1355.

17. Strauss I, Frolov V, Buchbut D, Gonen L, Maimon S. Critical appraisal of endovascular treatment of brain arteriovenous malformation using Onyx in a series of 92 consecutive patients. Acta Neurochir (Wien) 2013;155:611-617.

18. Valavanis A, Yasargil MG. The endovascular treatment of brain arteriovenous malformations. Adv Tech Stand Neurosurg 1998; 
24:131-214.

19. van Rooij WJ, Jacobs S, Sluzewski M, van der Pol B, Beute GN, Sprengers ME. Curative embolization of brain arteriovenous malformations with onyx: patient selection, embolization technique, and results. AJNR Am J Neuroradiol 2012;33:1299_
1304.

20. Westphal M, Grzyska U. Clinical significance of pedicle aneurysms on feeding vessels, especially those located in infratentorial arteriovenous malformations. J Neurosurg 2000;92:9951001. 